\title{
Escala Retrospectiva de Imprevisibilidade Familiar (Retro-FUS): Análise Rasch da versão Portuguesa
}

\author{
The Retrospective Family Unpredictability Scale (Retro-FUS): Portuguese \\ version Rasch analysis
}

\author{
José Pacheco Miguel*, Teresa Sousa Machado*, José Tomás da Silva*, Pedro Tiago Pereira* \\ * Universidade de Coimbra
}

\begin{abstract}
Resumo
O presente estudo analisa as propriedades psicométricas da Retrospective Family Unpredictability Scale (Retro-FUS) numa amostra de universitários portugueses, com o modelo de Rasch. Os resultados indicam que os itens ajustam a uma estrutura latente unidimensional, pressuposto requerido pelo modelo. A escala de resposta revelou funcionamento adequado, mas refinável, permitindo que os parâmetros de pessoas e itens fossem estimados com elevada precisão. Análises DIF confirmaram a inexistência de diferenças nos resultados relativas ao género. São discutidas as limitações do estudo e as implicações psicométricas dos resultados para futuras investigações.

Palavras chave: imprevisibilidade familiar, Retro-FUS, Rasch, DIF
\end{abstract}

\begin{abstract}
The present study analyzes the psychometric properties of the Retrospective Family Unpredictability Scale (Retro-FUS) in a sample of Portuguese university students with the Rasch model. The results indicated that scale items are well fitted to a latent unidimensional structure, as required by Rasch modeling. The response scale showed proper functioning, that can be refined, allowing people and item parameters to be estimated with high precision. DIF analyses confirmed no differences between genders in the results. Limitations of this study and the psychometric implications of its results are discussed, providing suggestions for future research.

Keywords: family unpredictability, Retro-FUS, Rasch, DIF
\end{abstract}

A imprevisibilidade familiar é definida por "inconsistent behaviors and regulatory system of the familiy" (Ross \& Hill, 2000, p.549). Há diferentes variáveis que podem ser responsáveis pela imprevisibilidade familiar, como sejam, a qualidade inconstante da educação e/ou inconsis- tente da disciplina, variações significativas nos recursos financeiros ou a falta de rituais nos horários das refeições. A literatura corrobora a noção de que a imprevisibilidade familiar induz stress e esgotamento nas crianças e nos pais, dificultando o estabelecimento de planos e a estru- turação do futuro. A imprevisibilidade familiar pode re- sultar de condições ambientais de risco (e.g., pobreza, guerra, emigração, desemprego) ou estar associada com stressores parentais como divórcio, alcoolismo e doenças. Estes stressores têm em comum o facto de todos eles irem comprometer as rotinas familiares que podem promover os resultados da previsibilidade familiar nas crianças. A actual situação socioeconómica portuguesa, caracterizada por instabilidade no emprego e diminuição nos salários, contribui para elevar os níveis de imprevisi- bilidade familiar, reforçando a tese acerca das relações entre estatuto económico e estrutura e qualidade de vida familiar e satisfação conjugal (Larson, Wilson \& Beley, 1994; Ross \& McDuff, 2008; Voydanoff, 2005). A impre- visibilidade está associada à disfunção familiar e a um acréscimo de comportamentos de risco infantis. Ausência de padrões estáveis de comportamentos adaptativos e dificuldade, ou impossibilidade dos pais monitorizarem o comportamento das crianças - que pode orientar o que e como as crianças deveriam fazer aumenta a probabilida- de estados mentais disruptivos e de atitudes problemáti- cas ao longo do desenvolvimento das crianças. A impre- visibilidade familiar está associada a uma cascata de comportamentos negativos crianças e adolescentes, tais como menor rendimento académico, absentismo infantil, gravidez indesejada, envolvimento em comportamentos anti-sociais e incapacidade de prevenir doenças comuns (Hill, Ross \& Low, 1997; Hill, Jenkins \& Farmer, 2008; Plunkett, Behnke, Sands \& Choi, 2009). O problema é que se não se acredita na possibilidade de se controlar o quotidiano, torna-se difícil acreditar na capacidade de se proporcionar o futuro.

A percepção de que o ambiente familiar é imprevisível remete para a construção de representações de controlo para ajustar e organizar acontecimentos (Howar- -Rodrigues \& Tokumaru, 2014). Por outro lado, como os modelos teóricos da vinculação têm sugerido, as rotinas familiares - ou previsibilidade - ao longo da infância promovem o desenvolvimento de modelos de relaciona- mentos adaptativos (permitindo o desenvolvimento de vinculações seguras) e selfs 
adaptativos (Bowlby, 1988; Bretherton \& Munholland, 2008). A mesma teoria esta- belece que a vinculação ambivalente insegura, e em maior grau, o padrão de vinculação desorganizado, está relacio- nado com a imprevisibilidade dos cuidados infantis. É a imprevisibilidade da sua vida que impede as crianças de construírem modelos internos de funcionamento seguros e normas de comportamento. A mesma imprevisibilidade irá perturbar o desenvolvimento adaptativo da planifi- cação do futuro, na medida em que essas crianças não podem confiar num passado imprevisível.

Considerando a transmissão intergeracional de compe- tências não cognitivas referidas na literatura (Vásquez \& Cruz, 2013), é expectável que a imprevisibilidade fami- liar também seja reproduzida em algumas das futuras fa- mílias destas crianças. É significativo notar que a invés- tigação revela, de forma consistente, uma correlação positiva entre vinculação segura e rotinas familiares previsíveis, (Laghi, D’Alessio, Pallini \& Baiocco, 2009).

A literatura relativa ao efeito do stresse na qualidade da vida, por exemplo, refere que entre os muitos aconteci- mentos e circunstâncias ameaçadoras da vida, os que se caracterizam por intensidade, duração e imprevisibilidade são os que tendem a ser os mais stressantes (Wolf, Noh, Fisman \& Speechley, 1989).

Tendo em consideração a importância do construto de imprevisibilidade familiar, bem como da Retrospective Family Unpredictability Scale (Retro-FUS) para a sua avaliação, associado ao facto de que os anteriores estudos com a versão Portuguesa da escala recorreram à teoria clássica dos testes (TCT), optou-se por avaliar agora as suas propriedades psicométricas no âmbito da teoria de resposta ao item (TRI). De forma específica, optou-se pelo modelo de Rasch que, pelas suas propriedades métricas, usa as respostas dos sujeitos como estatísticas suficientes para estimar os parâmetros relativos à dificuldade dos itens e ao nível de atributo (imprevisibili- dade familiar) dos sujeitos, de forma simultânea e inde- pendente (Wright \& Mok, 2004).

\section{Método}

Foram realizadas análises de Rasch, com o software Winsteps 3.73.0 (Linacre, 2011). Especificamente, dado o formato politómico invariante de todos os itens da escala, as estimativas dos parâmetros relativos aos itens, aos sujeitos e às categorias de resposta foram calculadas com o Rating Scale Model (RSM, Wright \& Masters, 1982). O RSM é uma extensão do modelo de Rasch para itens politómicos e que, segundo Linacre (2002), tem a seguinte formalização matemática:

$$
\log \left[P_{n i k} / P_{n i(k-1)}\right]=B_{n}-D_{i}-F_{k}
$$

$P_{\text {nik }}$ representa a probabilidade da pessoa $n$ responder na categoria $k$ do item $i, P_{n i(k-1)}$ é a probabilidade da pessoa $n$ responder na categoria $k-1$ do item $i, B_{n}$ é a medida da pessoa $n$ no traço avaliado, $D_{i}$ é a dificuldade do item $i$ e $F_{k}$ é a dificuldade do passo da categoria $k-1$ para a categoria $k$ (i.e., calibração do passo); esta calibração do passo $\left(F_{k}\right)$ é um limiar da escala de classifica- ção definido como sendo a localização correspondente à equiprobabilidade de observação das categorias adjacen- tes $k-1$ e $k$.

A opção pelo RSM explica-se pelas suas propriedades métricas que permitem que a comparação das pessoas seja independente dos itens usados para as medir e que a comparação dos itens seja invariante das pessoas usadas para os calibrar (objectividade específica), para além de transformar os dados ordinais relativos às respostas dos sujeitos numa escala intervalar (Wright \& Mok, 2004). Em termos práticos, ao apresentar estatísticas para o ajus- tamento da pessoa e do item, tem a vantagem de não requerer amostras grandes para estimar estes parâmetros. Permite ainda a determinação empírica da qualidade dis- criminativa das categorias nas escalas de resposta de tipo Likert (Bond \& Fox, 2007).

A avaliação da versão Portuguesa da Retro-FUS-P com o RSM centrou-se nos aspectos da validade de conteúdo, estrutural e substantiva, propostos por Wolfe \& Smith (2007), com base no trabalho de Messick (1995).

\section{Participantes}

Participaram no estudo 261 alunos dos $1^{\circ}$ e $2^{\circ}$ anos de diferentes cursos da Universidade de Coimbra. As idades variaram entre um mínimo de 18 e um máximo de 25 anos, correspondendo a uma média de 20.67 (DP $=$ 3.34). A distribuição é equilibrada quanto ao género, embora favoreça o sexo feminino $(52.8 \%)$. Não foi recolhida informação sobre a etnia por não ser uma variável rele- vante para os estudos realizados em Portugal (a maioria dos respondentes eram caucasianos europeus).

\section{Instrumentos}

Este estudo integrou um projecto de investigação que incluiu uma bateria de instrumentos para avaliar várias medidas psicológicas (perspectiva temporal de futuro, aspirações de carreira e satisfação com a vida), incluindo a imprevisibilidade familiar. Foi recolhida informação demográfica (sexo, idade, sucesso escolar e habilitações académicas dos pais).

A Retrospective Family Unpredictability Scale (RetroFUS; Ross \& McDuff, 2008) é uma medida de autorelato que avalia aspectos relacionados com a imprevisibilidade familiar. Os seus 28 itens proporcionam uma medida global do construto $(\alpha=.90)$ ou medidas em seis subescalas: refeições ( 5 itens, $\alpha=.83$ ), dinheiro ( 3 itens, $\alpha=.84$ ), afecto maternal ( 6 itens, $\alpha=.80$ ), afecto paternal (6 itens, $\alpha=.83$ ), disciplina maternal (4 itens, $\alpha=.85$ ), disciplina paternal (4 itens, $\alpha=.84$ ). A resposta é dada numa Likert de seis pontos de ancoragem ( 1 = Não se aplica nada, 2 = Aplica-se um pouco, 3 = Aplica-se moderadamente, $4=$ Aplica-se muito, 5 = Aplica-se imenso e $6=$ Não se aplica).

\section{Procedimento}

O estudo foi realizado no ano lectivo 2014/2015, em contexto de sala de aula, com uma duração aproximada de 25 minutos. Assegurou-se o consentimento informado dos participantes e garantiu-se a confidencialidade e o direito de abandono sem penalização. 


\section{Resultados}

A TRI, e por conseguinte o modelo de Rasch, assenta, entre outros, no princípio da unidimensionalidade; os itens de uma escala medem um único atributo, ou traço latente, de modo a que o desempenho do sujeito apenas é influenciado por um factor dominante (Bond \& Fox, 2007; Miguel, 2013). Na medida em que a Retro-FUS, apesar de avaliar dimensões independentes (Ross \& Hill, 2000), também proporciona uma medida global do cons- truto (Ross \& McDuff, 2008), a análise dos dados com o RSM começou por averiguar a dimensionalidade da escala como um todo, antes ainda de ser realizada de modo independente para cada uma das subescalas.

Para averiguar a validade estrutural, calculou-se a Análise de Componentes Principais (ACP) dos resíduos padronizados depois de controlar a dimensão Rasch, para determinar se os itens correspondem ao construto definido (Smith, 2004). À falta de consenso nos critérios para a presença de uma dimensão secundária (Chou \& Wang, 2010), optou-se por considerar um eigenvalue não superior 2.0 e uma variância explicada pelo primeiro componente dos resíduos superior a $20 \%$ como indicado- res da unidimensionalidade dos itens (Linacre, 2011).

A Análise de Componentes Principais dos Resíduos (ACPR) à FTPS-P, permite constatar que os dados parecem cumprir com o critério da unidimensionalidade, uma vez que o componente principal explica $35.3 \%$ ( $>$ $20 \%$ ) da variância total, embora o valor do eigenvalue para o primeiro contraste (4.6) exceda o limiar de aceitabilidade psicométrica (2.0) (Linacre, 2011), deixando em aberto a possibilidade dos resíduos padronizados poderem conter informação sistemática adicional (Fisher, 2007). Nesse sentido, calculou-se uma Análise Factorial Exploratória, Análise de Componentes Principais (ACP), com o SPSS. A Tabela 1 apresenta os itens extraídos, as saturações factoriais e as respectivas comunalidades, bem como o eigenvalue, a proporção de variância explicada e a consistência interna para o único componente extraído. Os resultados corroboram a tese da unidimensionalidade porque o eigenvalue do único componente extraído dupli- ca o valor do seguinte $(\lambda=3.459)$, critério indicador de um factor latente dominante em escalas multifactoriais (Reckase, 1979).
Tabela 1.

Retro-FUS: matriz de componentes

\begin{tabular}{|c|c|c|}
\hline Item & Componente & $h^{2}$ \\
\hline 1 & .445. & .198 \\
\hline 2 & .457 & .208 \\
\hline 3 & - & .054 \\
\hline 4 & .428 & .183 \\
\hline 5 & .476 & .227 \\
\hline 6 & - & .089 \\
\hline 7 & .366 & .134 \\
\hline 8 & .485 & .236 \\
\hline 9 & .532 & .283 \\
\hline 10 & .620 & .384 \\
\hline 11 & .594 & .353 \\
\hline 12 & .311 & .096 \\
\hline 13 & .460 & .212 \\
\hline 14 & .485 & ..235 \\
\hline 15 & .648 & .420 \\
\hline 16 & .625 & .390 \\
\hline 17 & - & .009 \\
\hline 18 & .595 & .354 \\
\hline 19 & .656 & .431 \\
\hline 20 & .668 & .446 \\
\hline 21 & .649 & .421 \\
\hline 22 & .505 & .255 \\
\hline 23 & .592 & .350 \\
\hline 24 & - & .074 \\
\hline 25 & .639 & .409 \\
\hline 26 & .589 & ,347 \\
\hline 27 & .321 & .103 \\
\hline 28 & - & .062 \\
\hline$\lambda$ & 6.964 & \\
\hline$\%$ & 24.87 & \\
\hline$\alpha$ & .89 & \\
\hline
\end{tabular}

As análises Rasch proporcionam indicadores para quantificar o ajustamento do modelo, estimar os parâme- tros do item e da pessoa e diagnosticar o funcionamento das categorias de resposta aos itens (Fox \& Jones, 1998). A Tabela 2 apresenta as estatísticas dos itens para o ajustamento (infit e outfit), a localização $\left(D_{i}\right)$ e o erro padrão $(E P)$ que permitem avaliar a validade de conteúdo da Retro-FUS, bem como as respectivas média $(M)$ e desvio padrão $(D P)$, para além dos coeficientes de validade estrutural (CE) da escala (Wolfe \& Smith, 2007). 
Tabela 2.

Propriedades psicométricas da Retro-FUS

\begin{tabular}{|c|c|c|c|c|c|c|}
\hline \multirow[b]{2}{*}{ Item } & \multicolumn{2}{|c|}{ MNSQ } & \multirow[b]{2}{*}{$D_{i}$} & \multirow[b]{2}{*}{$E P$} & \multirow[b]{2}{*}{$r_{p m}$} & \multirow[b]{2}{*}{$\mathrm{CE}$} \\
\hline & Infit & Outfit & & & & \\
\hline 1 & 1.06 & 1.06 & .20 & .06 & .46 & -.36 \\
\hline 2 & 1.07 & 1.08 & -.15 & .06 & .48 & -.43 \\
\hline 3 & 1.09 & 1.21 & -.12 & .06 & .27 & -.23 \\
\hline 4 & .96 & .96 & .15 & .06 & .44 & -.30 \\
\hline 5 & 1.02 & 1.05 & -.58 & .06 & .50 & -.40 \\
\hline 6 & 1.32 & 1.33 & .45 & .07 & .32 & .21 \\
\hline 7 & 1.19 & 1.19 & .27 & .06 & .37 & .14 \\
\hline 8 & .96 & .96 & .20 & .06 & .47 & -.11 \\
\hline 9 & .93 & .95 & .15 & .06 & .52 & -.29 \\
\hline 10 & .80 & .81 & -.55 & .06 & .57 & .33 \\
\hline 11 & .86 & .87 & -.47 & .06 & .56 & .34 \\
\hline 12 & .82 & .92 & .43 & .07 & .30 & -.07 \\
\hline 13 & 1.15 & 1.15 & -.54 & .06 & .47 & -.57 \\
\hline 14 & 1.13 & 1.17 & -.98 & .06 & .51 & -.53 \\
\hline 15 & .90 & .91 & -.13 & .06 & .57 & .44 \\
\hline 16 & .94 & .95 & -.12 & .06 & 57 & .54 \\
\hline 17 & 1.12 & 1.45 & .48 & .07 & .12 & -.09 \\
\hline 18 & .71 & .65 & .35 & .06 & .55 & -.29 \\
\hline 19 & .76 & .76 & .06 & .06 & .61 & -.35 \\
\hline 20 & .82 & .82 & -.37 & .06 & .60 & .57 \\
\hline 21 & .90 & .89 & -.26 & .06 & .59 & .63 \\
\hline 22 & 1.00 & 1.01 & -.33 & .06 & .50 & -.54 \\
\hline 23 & .90 & .92 & -.66 & .06 & .59 & -.50 \\
\hline 24 & 1.33 & 1.39 & .72 & .07 & .26 & .39 \\
\hline 25 & .96 & .93 & .21 & .06 & .56 & .56 \\
\hline 26 & .92 & .91 & .26 & .06 & .54 & .62 \\
\hline 27 & 1.20 & 1.28 & .54 & .07 & .30 & .26 \\
\hline 28 & 1.23 & 1.29 & .81 & .07 & .24 & .30 \\
\hline $\mathrm{M}$ & 1.00 & 1.03 & .00 & .06 & 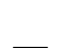 & \\
\hline DP & .16 & .19 & .44 & .00 & & \\
\hline
\end{tabular}

Em termos de validade de conteúdo, a Tabela 2 permite constatar que os valores médios de infit e outfit igualam ou quase coincidem com o valor esperado de 1.0 que indica um ajustamento perfeito dos itens; todos os itens da escala têm valores individuais no intervalo [.5 - 1.5] produtivo para a medida (Wright \& Linacre, 1994) o que indicia a inexistência de itens redundantes e a existência de homogeneidade entre todos eles. Este indicador da unidimensionallidade é confirmado por outros estimado- res da modelação Rasch que visam analisar o contributo dos itens na definição de um construto central para a estrutura interna da escala. As correlações ponto-medida $\left(r_{p m}\right)$, similares às correlações item-total da teoria clássi- ca dos testes (TCT), com valores entre .30 e .61 , sugerem a inexistência de ruído não modelado ou de dependência nos dados (Linacre, 2011), permitindo concluir que os itens, contribuem para definir um construto. Os valores inferiores a .30 correspondem aos itens que a ACP/TCT não encaixou na única dimensão extraída, à excepção do item 6 . Em termos dos $\mathrm{CE}$, apenas oito itens têm valores superiores a .40 e destes só dois têm valor superior a .60 .

Complementarmente, o erro-padrão dos itens, variando entre .06 e .07 indica que os itens mediram com elevada fiabilidade dentro da escala. A TRI disponibiliza ainda, em termos de fiabilidade da medida, dois índices para se examinar a proporção de variância dos itens e dos sujeitos não explicada pelo erro-padrão da medida, a item separation reliability (precisão de separação dos itens) e a person separation reliability (precisão de separação dos sujeitos); esta última é um valor homólogo ao coeficiente alfa (Prieto \& Delgado, 2003). Com .88 a precisão de separação dos sujeitos é boa (Fisher, 2007); a precisão de separação dos itens (.98) é excelente (Fisher, 2007).

A validade substantiva refere-se ao diagnóstico do fun- cionamento empírico das categorias da escala de respos- ta, com o propósito de determinar se estas funcionam em conformidade com aquilo que era esperado pelo autor quando desenvolveu os itens (Wolfe \& Smith, 2007). No contexto do RSM, Linacre (2002) propôs um conjunto de critérios para determinar a eficiência das categorias de resposta: (a) distribuição uniforme das frequências das respostas pelas diferentes categorias, com um mínimo de 10 observações em cada uma; (b) progressão monotónica da medida média obser- vada $-B_{n}-$ e das calibrações dos passos $-F_{k}-$ ao longo das categorias de resposta; e (c) outfit MNSQ, mais sen- sível que o infit a respostas não esperadas, das categorias de resposta inferior a 2.0.

Tabela 3.

Retro-FUS: estatísticas das categorias de resposta

\begin{tabular}{crrrrrrr}
\hline & \multicolumn{2}{c}{ Observada } & & \multicolumn{2}{c}{ MNSQ } & \\
\cline { 2 - 3 } Categoria & \multicolumn{1}{c}{$f_{i}$} & $\%$ & \multicolumn{1}{c}{$B_{n}$} & Infit & Outfit & $F_{k}$ \\
\hline 0 & 186 & 3 & -1.23 & 1.25 & 1.04 & - \\
1 & 2222 & 30 & -.35 & .90 & .92 & -3.52 \\
2 & 2025 & 28 & .12 & .96 & .98 & .00 \\
3 & 1544 & 21 & .46 & .93 & .98 & .53 \\
4 & 890 & 12 & .70 & 1.00 & 1.06 & 1.11 \\
5 & 441 & 6 & .89 & 1.11 & 1.24 & 1.54 \\
\hline
\end{tabular}

A Tabela 3 resume as estatísticas requeridas para avaliar em que medida as 6 categorias de resposta da Retro-FUS cumprem com estes critérios. A análise permite concluir que a estrutura da escala de resposta cumpre os critérios estipulados por Linacre (2002), uma vez que cada uma das categorias possui uma frequência observada de res- postas superior a 10 e um valor de outfit inferior a 2.0; para além disso, a medida média observada e a calibração dos passos também aumentam de forma monotónica ao longo das categorias, discriminando a amostra quanto ao atributo é medido.

O modelo de Rasch faz o escalonamento conjunto dos itens e dos sujeitos numa mesma dimensão (escala logit). Os parâmetros dos itens e dos sujeitos são expressos numa métrica comum, com as mesmas unidades (logits), numa escala de intervalos iguais, comparáveis entre si e localizados no mesmo contínuo (dimensão) (Embretson \& Reise, 2000). É desejável 
que as estimativas relativas ao atributo (pessoa) e à dificuldade (item) se sobreponham de forma substancial, para que o conjunto dos itens seja apropriado para a amostra. Sempre que a diferença entre as médias destas estimativas é inferior a 1 logit, significa que a informação contida nos itens permite discriminar as pessoas de forma precisa no construto que a escala se propõe medir (Bond \& Fox, 2007). No caso presente, o valor para essa diferenças é de apenas .13.

Foi ainda analisado o funcionamento diferencial dos itens (DIF) para avaliar a validade dos resultados da Retro-FUS-P relativamente ao género. Calculou-se a diferença padronizada entre as localizações dos parâmetros masculino e feminino, depois de se terem ajustado as possíveis diferenças relativas ao sexo na distribuição da imprevisibilidade familiar; usou-se o procedimento de Bonferroni que corrigiu o nível de significância em função do número de comparações $(.05 / 28)$ (Linacre, 2011). Seguindo este critério conservador, nenhum dos itens revelou localização significante superior a .50, valor estipulado por Wright \& Douglas (1975) como ponto de corte para o contraste DIF, nem no sexo masculino, nem no feminino. Conclui-se, portanto, que os itens da Retro- FUS têm funcionamento invariante quanto ao género.

\section{Discussão}

Globalmente, o estudo confirma que a Retro-FUS-P é um instrumento válido na avaliação da imprevisibilidade familiar dos alunos do ensino superior português. Os resultados validam a unidimensionalidade da escala; a utilização do modelo de Rasch, permite que os níveis do construto das pessoas e de dificuldade com que os itens o medem sejam hierarquizados num contínuo escalar para esta dimensão. Fá-lo com grau de precisão elevado que garante a adequação da escala de resposta e sem enviesa- mento quanto ao género.

A principal implicação deste estudo resulta, tal como os resultados comprovam, na demonstração da versão portuguesa como medida multidimensional intervalar. $\mathrm{O}$ ajustamento ao modelo de Rasch permite criar uma escala linear (logits) para itens e pessoas que proporciona valores expressos nas mesmas unidades. Ao demonstrar que os itens e a amostra de calibração são independentes, permitindo comparar o nível de imprevisibilidade fami- liar do sujeito directamente com o nível de dificuldade do item usado para a medir, confirma a existência de uma estruturas simples, essencial para a medição invariante, base para modelos de medida úteis (Wright \& Masters, 1982).

O estudo apresenta algumas limitações que importa superar numa próxima investigação, designadamente no que diz respeito à amostra que, embora reproduzisse as características demográficas da população visada, não é representativa da realidade Portuguesa. Para que os resultados possam ser generalizáveis importa que no futuro se recorra a métodos de amostragem aleatória.

\section{Referências}

Bond, M.H. \& Smith, P.B. (1996). Cross-cultural social and organizational psychology. Annual Review of Psychology, 47, 205-235.

Bowlby, J. (1988). A secure base. Clinical applications of attachment theory. London: Routledge.

Bond, T.G., \& Fox, C.M. (2007). Applying the Rasch model. Fundamental measurement in the human scien- ces (2nd ed.). Mahwah, NJ: Lawrence Erlbaum.

Bretherton, I. \& Munholland, K.A. (2008). Internal working models in attachment relationships: Elaborating a central construct in attachment theory. In J. Cassidy \& P.A. Shaver (Eds.), Handbook of attachment: Theory, research and clinical applications (2nd ed., pp. 102-127). New York: Guilford Press.

Chou, Y.-T., \& Wang, W.-C. (2010). Checking dimensionality in item response models with principal component analysis on standardized residual. Educational and Psychological Measurement, 70, 717-731. http:// dx.doi.org/10.1177/0013164410379322

Embretson, S.E. \& Reise, S.P. (2000). Item Response Theory for Psychologists. Mahwah, NJ: Lawrence Erlbaum Associates.

Fisher, W.P. Jr. (2007). Rating scale instrument quality criteria. Rasch Measurement Transactions, 21, p. 1095.

Fox, C.M., \& Jones, J.A. (1998). Uses of Rasch modeling in counseling psychology research. Journal of Counse- ling Psychology, 45, 30-45. http://dx.doi.org/10.1037// 0022-0167.45.1.30

Hill, E.M. Jenkins, J. \& Farmer, L. (2008). Family unpre- dictability, future discounting, and risk taking. The Journal of Socio-Economics. 37, 200-216.

Hill, E.M., Ross, L.T. \& Low, B.S. (1997). The role of future unpredictability in human risk-taking. Human Nature, 8(4), 287-325.

Laghi,F., D’Alessio, M., Pallini, S. \& Baiocco, R. (2009). Attachment representations in adolescence. Social Indicators Research, 90(2), 181-194.

Larson, J.H., Wilson, S.M. \& Beley, R. (1994). The impact of job insecurity on marital and family relation- ships. Family Relations, 43(2), 138-143.

Linacre, J.M. (2002). Optimizing rating scale category effectiveness. Journal of Applied Measurement, 3(1), 85-106.

Linacre, J.M. (2011). Winsteps Rasch measurement computer program, version 3.73.0. [Computer program] Chicago, Il: Winsteps.com.

Messick, S. (1995). Validity of psychological assessment: Validation of inferences from persons' responses and performances as scientific inquiry into score meaning. American Psychologist, 50, 741-749. http://dx.doi.org/ 10.1037//0003-066x.50.9.741

Miguel, J.P. (2013). TRI: representação e utilidade do modelo logístico de traço latente na psicometria actual. Dissertação de Doutoramento, Faculdade de Psicologia e de Ciências da Educação, Universidade de Coimbra, Coimbra, Portugal

Plunkett,S.W., Behnke, A.O., Sands, T. \& Choi, B.Y. (2009). Adolescents' reports of parental engagement 
and academic achievement in immigrant families. Journal of Youth and Adolescence, 38, 257-268.

Prieto, G. \& Delgado, A.R. (2003). Analisis de un test mediante el modelo de Rasch. Psicothema, 15(1), 94-100.

Reckase, M. (1979). Unifactor latent trait models applied to multifactor tests: results and implications. Journal of Educational Statistics, 4, 207-230.

Ross, L.T. \& Hill, E.M. (2000). The Family Unpredictabili- ty Scale: Reliability and validity. Journal of Marriage and Family, 62(2), 549-562.

Ross, L.T. \& McDuff, (2008). The retrospective family unpredictability scale: Reliability and validity. Journal of Child and Family Studies, 17(1), 13-27.

Smith Jr, E.V. (2004). Detecting and evaluating the impact of multidimensionality using item fit statistics and PCA of residuals. In: E.V. Smith Jr \& R.M. Smith (Eds.), Introduction to Rasch measurement (pp. 575-600). Maple Grove, MN: JAM Press.

Vásquez, A. \& Cruz, O. (2013). "Star thinking what are you to bewhen adult!" The relationship between paren- tal time orientation and the emergence of episodic fore- sight in preschool aged children. In M.P. Paixão \& J.T. Silva (Coord.), International Studies in Time Perspec- tive. Coimbra: IUC.

Voydanoff, P. (2005). Toward a conceptualization of perceived work-family balance: A demands and resour- ce approach. Journal of Marriage and Family, 67(4), 822-836.

Wright, B.D., \& Masters, G.N. (1982). Rating scale analysis. Chicago, Il: MESA Press.

Wolfe, E.W., \& Smith Jr, E.V. (2007). Instrument deve- lopment tools and activities for measure validation using Rasch models: Part II - Validation activities. In E.V. Smith Jr \& R.M. Smith (Eds.), Rasch measure- ment: Advanced and specialized applications (pp. 243-290). Maple Grove, MN: JAM Press.

Wolf, L.C., Noh, S., Fisman, S.N. \& Speechley, M. (1989). Brief report: Psychological effects of parenting stress on parents of autistic children. Journal of Autism and Developmental Disorders, 19(1), 157-166.

Wright, BD., \& Linacre, J.M. (1994). Reasonable mean-square fit values. Rasch Measurement Transactions, 8, p. 370.

Wright, B. D. \& Mok, M.M. (2004). An overview of the family of Rasch measurement models. In E.V. Smith Jr \& R.M. Smith (Eds.), Introduction to de Rasch Measurement (pp. 1-24). Maple Grove, MN: JAM Press.

\section{Agradecimientos}

Investigação realizada no âmbito do Laboratório de Psicometria da Faculdade de Psicologia da Universidade de Coimbra 